\title{
L’analyse du texte littéraire assistée par ordinateur : essai d'illustration avec Regards et jeux dans l'espace, de Saint-Denys Garneau, traité avec le logiciel SATO Computer-assisted Analysis of a Literary Document: Test Case of Regards et jeux dans l'espace by Saint-Denys Garneau, Using SATO \\ El análisis del texto literario asistido por computadora: ejemplo con Regards et jeux dans l'espace (Miradas y juegos espaciales), de Saint-Denys Garneau, tratado con el programa SATO
}

\author{
Suzanne Bertrand-Gastaldy et Paul Marchand
}

Volume 45, numéro 2, avril-juin 1999

URI : https://id.erudit.org/iderudit/1032764ar

DOI : https://doi.org/10.7202/1032764ar

Aller au sommaire du numéro

\section{Éditeur(s)}

Association pour l'avancement des sciences et des techniques de la documentation (ASTED)

ISSN

0315-2340 (imprimé)

2291-8949 (numérique)

Découvrir la revue

Citer cet article

Bertrand-Gastaldy, S. \& Marchand, P. (1999). L’analyse du texte littéraire assistée par ordinateur : essai d'illustration avec Regards et jeux dans l'espace, de Saint-Denys Garneau, traité avec le logiciel SATO. Documentation et bibliothèques, 45(2), 55-66. https://doi.org/10.7202/1032764ar

\section{Résumé de l'article}

Les textes de tous genres se retrouvent en nombre grandissant sur des supports électroniques et l'ordinateur peut être mis à contribution pour aider à faire leur analyse. Les textes littéraires, avec leurs particularités, n’échappent pas à cette tendance que les chercheurs accueillent avec scepticisme ou intérêt. Le développement des bibliothèques électroniques incite les spécialistes en information documentaire à étendre leur expertise et à diversifier leurs services pour des clientèles mieux informées et plus exigeantes. Une recherche exploratoire menée à l'aide du logiciel SATO (Système d'analyse de texte par ordinateur) sur un recueil de poèmes, Regards et jeux dans l'espace, de Saint-Denys Garneau, vise à illustrer certaines des données et des interprétations qui peuvent être tirées, selon diverses approches, de traitements statistiques et sémantiques. Des études de plus grande envergure portant sur un ensemble de corpus peuvent être envisagées, renouvelant certaines problématiques littéraires.
Tous droits réservés (c) Association pour l'avancement des sciences et des techniques de la documentation (ASTED), 1999
Ce document est protégé par la loi sur le droit d'auteur. L'utilisation des services d’Érudit (y compris la reproduction) est assujettie à sa politique d'utilisation que vous pouvez consulter en ligne. 


\title{
L'analyse du texte littéraire assistée par ordinateur: essai d'illustration avec Regards et jeux dans l'espace, de Saint-Denys Garneau, traité avec le logiciel SATO
}

\author{
Suzanne Bertrand-Gastaldy \\ Professeure titulaire \\ École de bibliothéconomie et des sciences de l'information, Université de Montréal \\ Paul Marchand \\ Bibliothécaire \\ École de technologie supérieure, Université du Québec*
}

Les textes de tous genres se retrouvent en nombre grandissant sur des supports électroniques et l'ordinateur peut être mis à contribution pour aider à faire leur analyse. Les textes littéraires, avec leurs particularités, n'échappent pas à cette tendance que les chercheurs accueillent avec scepticisme ou intérêt. Le développement des bibliothèques électroniques incite les spécialistes en information documentaire à étendre leur expertise et à diversifier leurs services pour des clientèles mieux informées et plus exigeantes. Une recherche exploratoire menée à l'aide du logiciel SATO (Système d'analyse de texte par ordinateur) sur un recueil de poèmes, Regards et jeux dans l'espace, de Saint-Denys Garneau, vise à illustrer certaines des données et des interprétations qui peuvent être tirées, selon diverses approches, de traitements statistiques et sémantiques. Des études de plus grande envergure portant sur un ensemble de corpus peuvent être envisagées, renouvelant certaines problématiques littéraires.

\section{Computer-assisted Analysis of a Literary Document: Test Case of Regards et jeux dans l'espace by Saint-Denys Garneau, Using SATO}

\begin{abstract}
A growing number of different types of documents are currently available electronically and can be analysed by computer. Literary texts and their specific characteristics are also being analysed in this manner and researchers greet this development with interest or scepticism. The development of electronic libraries invites information specialists to apply their expertise and to diversify their services to meet the new needs of better informed clients. $A$ test case using the SATO software (Système d'analyse de texte par ordinateur) was carried out on a collection of poems, Regards et jeux dans l'espace by Saint-Denys Garneau; the intended purpose of this trial was to highlight certain facts and their interpretations using different approaches, varying from statistical to semantic analyses. Large-scale studies using the complete works of a author are possible, renewing to the study of certains types of literary issues.
\end{abstract}

EI análisis del texto literario asistido por computadora: ejemplo con Regards et jeux dans l'espace (Miradas y juegos espaciales), de Saint-Denys Garneau, tratado con el programa SATO

Cada vez más se encuentra todo tipo de textos en forma electrónica y la computadora puede servir para ayudar a analizarlos. Los textos literarios, con sus particularidades, siguen esta misma tendencia, que los investigadores reciben con escepticismo o interés. La creación de bibliotecas electrónicas incita a los especialistas en información documental a ampliar su pericia y a diversificar sus servicios para las clientelas mejor informadas y más exigentes. Una investigación piloto, realizada con la ayuda del programa SATO (Sistema de análisis de texto por ordenador), de una compilación de poemas, Regards et jeux dans l'espace, de Saint-Denys Garneau, trata de ilustrar ciertos datos e interpretaciones que pueden extraerse, conforme a diversos enfoques, del uso de las estadísticas y de la semántica. Es posible prever estudios de mayor envergadura hechos con un corpus variado, que renovarán ciertas problemáticas literarias.
L'ordinateur apporte des possibilités nouvelles et différentes pour analyser des textes disponibles en nombre grandissant sur support électronique. Les particularités des textes littéraires suscitent nombre de questions sur l'utilité de l'informatique dans les processus de lecture réputés pour être cognitivement complexes et ouverts. Afin d'expérimenter le genre de démarches possibles, nous avons testé quelques-unes des fonctionnalités du logiciel SATO (Système d'analyse de texte par ordinateur) ${ }^{1}$ sur le recueil Regards et jeux dans l'espace, de Saint-Denys Garneau, que

\footnotetext{
Cette recherche a été rendue possible grâce à un programme de mobilité des professionnels du réseau de l'Université du Québec.

Note de la rédaction: Les auteurs ont conservé le point comme signe décimal dans la présentation de leurs résultats. Des néologismes sont employés pour identifier certains concepts.

1. Ce logiciel, développé par François Daoust, est employé en sciences de l'information principalement pour le contrôle du vocabulaire et l'indexation assistés par ordinateur (voir Bertrand-Gastaldy 1994; Bertrand-Gastaldy et Pagola 1992) ainsi que dans d'autres disciplines qui font un large usage des textes, comme la linguistique ou la sociologie. Lors du congrès de l'ASTED de 1995, notamment, Guy Bertrand présentait ce logiciel comme un exemple d'intelligence numérique.
} 
nous avons choisi comme corpus d'expérimentation. La souplesse du logiciel2 a été exploitée pour le codage, le traitement et la comparaison de plusieurs types d'unités lexicales et textuelles. Une masse importante de données en a découlé et nous nous sommes fixés comme objectif, après avoir sélectionné quelques résultats, d'illustrer le genre d'information et le type d'interprétation qui sont en jeu. Des traitements statistiques et sémantiques appliqués au vocabulaire du recueil en entier et de ses divisions en suites et en poèmes, ainsi qu'une catégorisation à partir des thèmes de la vie et de la mort permettent des analyses de plus en plus fines des thèmes développés et même de l'architecture de l'œuvre. Finalement, des approches sociocritiques, de même que des approches d'inspiration psychanalytique et intertextuelle du texte littéraire sont esquissées. Ainsi, il s'avère possible de prendre en considération les multiples facettes et la complexité du texte littéraire, sans courir le danger de réduire sa lecture à des décomptes informatiques simplistes, le spécialiste restant toujours maître de l'instrument qu'il met au service de ses savoirs et de ses hypothèses.

\section{Le rôle grandissant de l'informatique dans les études littéraires}

Le recours à l'informatique dans les études littéraires devient quasi incontournable, ne serait-ce que parce que de plus en plus de textes littéraires appartenant au domaine public sont disponibles sur support électronique, soit sur disquettes, sur cédéroms et surtout dans Internet. La production provient, dans une faible proportion (11\% selon Gaunt 1995), d'éditeurs commerciaux comme les Éditions ILIAS ou Larousse en France et, dans une grande mesure, de chercheurs, de bibliothèques, de musées et de centres de recherche. Ainsi, pour les œuvres de langue française, il est possible, moyennant un abonnement, de consulter FRANTEXT ou son site miroir à l'Université de Chicago, ARTFL (American and French Research on the Treasury of the French Language) qui donnent accès aux quelque 3500 textes constituant le Trésor de la langue française (TLF) réunis par l'INALF (Institut national de la langue française). Pour l'anglais, le site le plus connu est Oxford Text Archives (OTA) qui, parmi ses 25000 textes, en contient quelques-uns en langue française. D'autres sites francophones sont également disponibles comme celui de l'Association des bibliophiles universels $(A B U)$ en France et ClicNet aux États-Unis. Plusieurs monographies, des périodiques spécialisés tels que Computers and the Humanities, Literary and Linguistic Computing, ou encore le double numéro de la revue torontoise Texte portant sur le texte et l'informatique paru en 1993, donnent une bonne idée de l'évolution du lien entre la littérature et l'informatique. Selon les données de Lenoble et Sabourin (1993), la production de la recherche impliquant la poésie figure parmi les plus importantes avec celles classifiées dans la stylistique, la fiction et la concordance; c'est aux États-Unis (48\%) et en France (11\%) que les analyses computationnelles se rapportant à ce genre littéraire sont les plus nombreuses.

Les discussions sur la liste SATOR-L et le projet de coopération universitaire «Application de la technologie informatique au traitement du texte narratif " constituent une autre manifestation de l'effervescence engendrée par les possibilités du traitement électronique. II s'agit d'introduire l'outil informatique dans le domaine des études littéraires et, plus particulièrement, dans celui de l'analyse du texte narratif, en ce qui a trait au repérage et au traitement des unités narratives de base récurrentes appelées «topoi »; ceux-ci sont saisis dans un thésaurus à l'aide du logiciel «Toposator» élaboré par l'équipe interdisciplinaire de la Société d'Analyse de la Topique Romanesque (SATOR) du Centre d'études sur le XVIIIe siècle de I'Université Paul Valéry-Montpellier III. Le 13 décembre 1998, un message diffusé par Michel Lemaire sur la liste de discussion BALZAC-L annonçait le lancement, par un groupe de recherche de l'Université d'Ottawa, du projet «Recherche littéraire et informatique » dont l'objectif essentiellement épistémologique est «de faire la critique des projets de recherche littéraire assistée par ordinateur passés et présents, d'analyser les concepts, les procédés, les instruments développés dans ces recherches, d'imaginer de nouvelles voies, de nouvelles façons de faire, de nouveaux types de recherche".

Les spécialistes de la documentation ne sont pas indifférents au traitement du texte littéraire électronique. En 1994, à l'École de bibliothéconomie et des sciences de l'information de l'Université d'Illinois, la rencontre de la $31^{e}$ Annual Clinic on Library Applications of Data Processing avait pour thème: "Literary Texts in an Electronic Age: Scholarly Implications and Library Services". Cet événement avait attiré plusieurs bibliothécaires aussi bien dans l'assistance que parmi les conférenciers. En revanche, l'appel de communications pour le congrès conjoint international 1998 de l'Association for Literary and Linguistic Computing et de l'Association for Computers and Humanities, signalait le peu de contributions en provenance de la bibliothéconomie dans les colloques précédents. C'est dans ce contexte de croisement entre disciplines que, profitant de la complémentarité de nos formations, nous avons entrepris l'analyse du recueil de Saint-Denys Garneau.

Les chercheurs en études littéraires ont des avis divergents sur l'utilité de l'informatique en littérature. Plusieurs soulignent que les approches informatiques relèvent d'une conception simpliste de ce qu'est un texte, envisagé comme une succession de chaînes de caractères séparés par des espaces blancs ou des caractères délimiteurs (ce qui, en passant, ne diffère pas de la plupart des systèmes de traitement et de repérage de l'information textuelle). Ils leur reprochent de ne pas permettre d'en appréhender les significations symboliques (Vuillemin 1993), les moyens d'expression comme les images, figures, conventions, ou les entités d'ordre supérieur comme l'intrigue ou l'atmosphère (Fortier 1995). Aux chercheurs qui, pour la plupart, s'appuient sur des calculs de fréquences de mots, il est facile d'objecter qu'une fréquence en ellemême n'a jamais rien prouvé, que «bien des phénomènes textuels sont créés par un nombre réduit d'occurrences sans signification statistique et qu'une petite différence quantitative peut déclencher d'importants effets de lecture" (Hamm et Lessard 1993, 264). Selon certains, les analyses automatiques ne révèlent pas grand chose d'autre qui n'était déjà connu ou qu'une lecture attentive peut mettre au jour (Olsen 1993). Lenoble (1993, 55 et $56)$, de son côté, rappelle que «le lien au texte électronique est empreint des limita-

2. SATO, par sa flexibilité, differe d'autres logiciels qui obligent à un parcours prédéterminé du texte. 
tions et des présupposés théoriques des programmes d'accès", forçant les communautés de lecteurs à poser sur lui «un regard étrangement balisé».

Par contre, l'ordinateur peut devenir l'allié de qui sait l'utiliser comme un outil auxiliaire pour la collecte et le traitement des données, capable de lui fournir un relevé exhaustif et non biaisé sur la distribution du vocabulaire révélatrice de l'importance de certaines thématiques. Les balises additionnelles ainsi obtenues peuvent assurer la solidité des interprétations du chercheur en le gardant d'une tendance naturelle à l'impressionnisme qui le conduit à surestimer les passages susceptibles de soutenir ses hypothèses (Taylor 1993). Les méthodes quantitatives doublées de mécanismes de catégorisation sémantique (Fortier 1995; Laffal 1995) s'avèrent fructueuses non seulement dans l'étude des thèmes d'une œuvre, mais aussi dans l'observation de l'évolution d'un thème au travers de plusieurs œuvres d'un même auteur ou de plusieurs auteurs. C'est ainsi que le thésaurus thématique des sentiments actuellement développé par l'INALF «permettra [...] de répondre instantanément à des questions du type les mots du thème de la «jalousie» chez Proust; le champ sémantique de l'«ennui» chez Baudelaire»3. Rastier $(1995,7)$ souligne que, «comme l'expansion des banques de données textuelles suscite des besoins croissants, l'enjeu d'une thématique est considérable, pour l'indexation comme pour l'exploitation des textes". Les recherches en littérature pour essayer de circonscrire ce qu'est un thème ( Fortier 1995; Martin 1993, 1995; Rastier 1989; Wooldridge 1993) devraient, en effet, bénéficier aussi aux sciences de l'information, dont une grande part des activités consiste à extraire le sujet - le thème ou l'aboutness - des textes afin d'en faciliter le repérage.

Des auteurs comme Olsen (1993) incitent leurs collègues à ne pas se cantonner dans l'étude de phénomènes littéraires pour lesquels les capacités de l'ordinateur sont trop limitées, mais à exploiter sa puissance de stockage et de traitement pour explorer plutôt des questions nouvelles, à l'instar des chercheurs en sciences sociales qui ont su développer des méthodes novatrices orientées, par exemple, vers l'intertextualité et la sémiotique sociale à travers l'analyse de gran- des quantités de textes de la même période ou distribués dans le temps. L'électronique change, en effet, les rapports entre le lecteur et le texte: elle donne accès «à des constructions ou à des déconstructions» (Olivier 1993, 295), elle l'immerge dans des ensembles textuels plus larges, l'ouvrant «aux possibilités d'interrelation de ses propres composantes avec d'autres parties de l'ensemble». (Lenoble 1993, 53)

Les chercheurs les plus optimistes voient dans le nouveau support l'occasion de se libérer de la contrainte des «lectures toujours partielles, puisque linéaires» qu'impose l'imprimé ainsi que la possibilité d'effectuer des «lectures verticales qui traversent tout le texte» (Wooldridge 1993, 275). Loin de souffrir de l'enfermement de parcours soi-disant préprogrammés, ils relèvent la multitude de concordances virtuelles qu'ils peuvent définir eux-mêmes en fonction de leurs hypothèses et besoins: formes simples ou lemmatisées, familles lexicales, sémantiques, syntagmatiques, thématiques, longueur variable des contextes, mesures statistiques d'occurrences, de cooccurrences, distribution, restriction par champs, etc. (Wooldridge 1993). Le choix est large, offrant plusieurs points de vue (Rastier 1995) et de nouveaux moyens d'accès au texte qui font se côtoyer vision macroscopique et vision microscopique (Hamm et Lessard 1993). Pour peu que la macroinformatique se double d'outils microinformatiques d'étiquetage dynamique, l'ordinateur peut accompagner la lecture, à mi-chemin entre l'analyse quantitative et l'analyse qualitative (Teil 1994). «Support sur lequel s'inscrit un nombre non défini de lectures possibles, le rôle de l'informatique consisterait à donner au chercheur les moyens d'enregistrer sur le texte le produit de sa lecture, de tisser des liens entre des phénomènes apparentés, selon l'optique qu'il a adoptée», font remarquer Hamm et Lessard (1993, 273).

C'est parce que le logiciel SATO, au cours d'expérimentations antérieures portant sur des opérations documentaires, nous a paru posséder un peu de ces caractéristiques que nous avons voulu tester son potentiel pour l'analyse de textes poétiques.

\section{Le corpus d'expérimentation}

Le corpus d'expérimentation choisi, Regards et jeux dans l'espace, est une œuvre tombée dans le domaine public en 1993. II présente un total de 28 poèmes répartis en huit divisions d'inégale longueur, en l'occurrence sept suites et un poème de conclusion: Jeux, Enfants, Esquisses en plein air, Deux paysages, De gris en plus noir, Faction, Sans titre, Accompagnement.

Le texte a été saisi au moyen d'un logiciel de traitement de texte à partir de l'édition critique de Brault et Lacroix (voir Garneau 1971) avant d'être soumis à divers traitements et analyses dans SATO.

\section{Quelques résultats sur le lexique d'un point de vue général}

Des données quantitatives générales sont d'emblée accessibles avec un logiciel comme SATO, à la fois sur le texte et sur le lexique. Ainsi, Regards et jeux dans l'espace est constitué de 4305 occurrences (4 395 si l'on compte les signes de ponctuation) et de 1202 formes lexicales non lemmatisées. Le lexique est formé de 8630 caractères. La longueur moyenne des mots est de 4.1 caractères et ce sont les mots de deux caractères qui sont les plus nombreux (29\%).

Un simple examen du lexique, pourvu qu'il puisse s'effectuer sous différents angles, à la fois pour l'ensemble du corpus et pour ses subdivisions, est susceptible de fournir une grande richesse d'information au lecteur doté de suffisamment de connaissances pour faire parler les signes. En ce sens, nous avons lu beaucoup d'études ayant déjà porté sur le recueil et nous avons pu constater que quelques-unes d'entre elles ont manifesté un intérêt certain pour des considérations lexicales et quantitatives (Giguère 1973; Melançon, Robert 1994; Ouellet 1994; Smoje 1984-85). L'examen du lexique a été effectué pour tout le corpus, pour chaque suite et pour chaque poème.

\footnotetext{
3. Explications fournies à l'adresse suivante: http://www.ciril.fr/INALF/inalf.presentation/frantext.htm.
} 


\section{Examen du lexique du corpus dans son ensemble}

Le lexique peut être édité selon divers tris. Le tri par fréquence décroissante a montré, par exemple, que le mot "comme" revient à 38 reprises $(0.86 \%)^{4}$, ouvrant ainsi une voie à l'analyse de la comparaison et même de la comparaison hypothétique puisque "comme " s'accompagne quatre fois de «si». D'autre part, nous avons noté une participation plus importante des marqueurs de l'espace («dans", avec 60 occurrences; «sur », 32; "sous", 8; etc.) par rapport à ceux du temps ("quand", 15 ; «puis", 5 ; «avant », 4 ; etc.): en tout, nous avons dénombré 138 occurrences pour les premiers contre 42 pour les seconds. Après les formes fonctionnelles dans le lexique viennent les mots-thèmes, ceux qui sont « les plus employés par un auteur; ceux par lesquels se motive la pensée" (Guiraud, voir Guiraud et Kuentz 1975). Le mot-thème "mort» domine avec 20 occurrences $(0.46 \%)$. Il est suivi, avec 18 occurrences $(0.41 \%)$, de "ciel » et de "yeux", et, avec 17 occurrences $(0.39 \%)$, de "vent", etc. Ces trois derniers mots-thèmes ainsi que plusieurs occurrences de lexèmes liés aux notions de regard, de jeu et d'espace sont en relation sémantique avec le titre du recueil qui joue alors le rôle de métatexte.

De la même façon que le titre tend à orienter préalablement la lecture, le ou les mots-thèmes peuvent faire office de paralexique susceptible de guider les analyses, comme nous le verrons plus loin à propos des regroupements du vocabulaire auxquels nous avons procédé en fonction du thème de la mort et de son opposé, le thème de la vie.

\section{Examen du lexique de chaque suite et leur relation au lexique global}

L'intérêt des études lexicométriques réside dans la comparaison; une démarche visant à rapprocher diverses suites du recueil a permis quelques constats aussi bien sur des formes fonctionnelles que sur des formes dites de signification. En voici quelques exemples.

La présence de 14 occurrences de «pas» dans la première suite $(1.65 \%)$ a d'abord attiré notre attention; cela représente plus du quart de la fréquence de ce mot dans le recueil entier où il revient 40 fois $(0.91 \%)$. Par surcroît, $23(0.52 \%)$ occurrences de la forme lexicale "sans", signe du privatif, soit le quart de toutes ses occurrences dans le recueil, se mêlent à ce portrait. Pourquoi d'entrée de jeu le poète s'exprime-t-il ou désigne-t-il les choses sur un mode dénégatif ?5 Voilà un exemple de piste pour l'analyse. $\mathrm{Ce}$ pendant, puisque le «domaine » de la première suite Jeux représente, en nombre d'occurrences, un peu moins de $20 \%$ de l'ensemble du recueil, nous pouvons conclure que le mode dénégatif ou privatif ne se localise pas uniquement au début de l'œuvre: D'ailleurs, on retrouve plusieurs lexèmes formés avec les préfixes 6 «il-», «im-» ou «in-»: «illimité», «imperceptible», «impondérable», «inattentive», «intolérable», etc., dans le lexique global.

Justement, c'est sous le titre significatif de Saint-Denys Garneau ou la vie impossible que Hénault, en 1969, nous confie que "vivre, c'est aussi un acte poétique, dans la mesure où le poète chante et enchante le réel", mais pour certains poètes, “les purs" précise-t-il, l'insertion dans la vie est difficile. "lly en a qui se retirent dans leurs châteaux de songes, pour y mourir", écrit-il. Et il énonce à propos de Garneau: «De l'enfance à la mort, il me semble qu'il n'y ait [...] qu'un tout petit intermède, un décor planté de saules et d'ormes calmes, un paysage à deux dimensions, dans lequel il ne peut pénétrer, mais qu'il décrit avec toute la sensibilité d'un aquarelliste.» (p. 483)

Des suites sont clairement en rapport avec le titre Regards et jeux dans l'espace. Avec respectivement $6(0.71 \%)$ et $8(0.72 \%)$ occurrences, soit 14 sur un total de 18 pour l'ensemble du recueil, "yeux" revient à deux reprises comme mot-thème dominant de deux suites, la première et la septième qui représentent les plus fortes densités lexicales de l'architecture de l'œurre. Dans la première suite se concentrent 5 occurrences de « regard $»\{0.59 \% ; 0.16 \%\}$ sur les 15 occurrences des formes flexionnelles de « regard » et « regarder » présentes dans le recueil, 5 de «voir » $\{0.59 \% ; 0.25 \%\}, 4$ de "jouer" $\{0.47 \% ; 0.11 \%\}$ et 3 de «espace $»\{0.35 \% ; 0.14 \%\}$.

Nous avons examiné les marqueurs pronominaux du sujet de l'énonciation. La sommation des occurrences de «je», «j”» et «moi » dans la séquence des divisions évolue de la façon suivante: $9(1.07 \%)$, $0.2(0.44 \%), 0.4(0.84 \%), 4(0.70 \%), 22$ $(1.98 \%)$ et $15(8.93 \%)$, pour un total de 56 occurrences $(1.27 \%)$ dont $66 \%$ se focalisent dans les deux dernières suites. La présence de ces pronoms s'accentue donc au fur et à mesure que l'on chemine vers la fin du recueil, comme dans un mouvement d'introspection ou d'intériorisation. Cela va de pair avec l'espace poétique qui passe du paysage ou des Esquisses en plein air à une maison fermée et à une chambre, puis à cette fameuse petite prison d'os: «Je suis une cage d'oiseau / Une cage d'os". (p. 33)

La distance est une opération de SATO qui "permet de comparer statistiquement les lexiques de deux sous-textes quelconques d'un corpus [...]. Plus la distance calculée [...] est grande, plus les deux sous-textes diffèrent dans l'utilisation d'un vocabulaire désigné [...]" (Daoust 1996, 88). Elle a été calculée entre chaque suite et l'ensemble des autres, et entre chaque suite et chacune des autres. C'est le lexique du poème de conclusion Accompagnement, considéré ici comme une division comparable aux suites, qui présente la plus grande originalité sur le plan lexical, et cela par rapport aux divisions en suites prises ensemble $(30.09)^{8}$ ou isolément, et c'est le lexème «joie » $\{7.54\}^{9}$ qui en est le premier responsable. Ce même lexème se révèle être le mot-thème de la dernière division du recueil. Vient au deuxième rang relativement à l'ensemble la deuxième suite, intitulée Enfants (24.57), avec le pronom «il » comme unité dominante $\{5.95\}$, pronom

4. La fréquence relative est entre parenthèses.

5. Il faut préciser que l'analyse strictement automatique doit nous porter à rester vigilants en face du caractère ambigu de certains signes pris hors contexte. Le sens de l'adverbe «pas » se distingue de celui de la forme nominale «pas» présente dans le poème final Accompagnement: «J'entends mon pas en joie qui marche à côté de moi / [...] Je ne puis pas mettre mes pieds dans ces pas-là». (p. 34)

6. C'est donc dire qu'avec SATO nous sommes allés en deçà des mots au complet pour analyser des éléments morphologiques.

7. Entre les accolades, le premier chiffre représente la fréquence relative pour la suite et le deuxième chiffre se rapporte à la fréquence relative de la forme lexicale dans tout le recueil.

8. Le chiffre quantifiant la distance est entre parenthèses.

9. SATO offre une donnée quantitative relative à l'unité dominante expliquant la distance: le chiffre entre accolades est en rapport avec l'ensemble des divisions. 
que l'on peut opposer à celui de la première personne qui ressort à la fin du recueil. C'est la septième suite qui, avec le plus grand nombre d'occurrences (près de $26 \%$ du recueil), démontre le moins d'originalité lexicale relativement à l'ensemble des autres (13.93), ce qui est, pour ainsi dire, en accord avec son titre: Sans titre.

\section{Examen du lexique de poèmes en particulier}

Avec des regroupements sémantiques de lexèmes, nous avons étendu l'analyse jusque dans les poèmes en particulier, comme l'illustrent ces deux exemples du vocabulaire de l'eau et du feu.

Pins à contre-jour, le dernier poème de la troisième suite, est placé sous le signe de l'eau. En plus de présenter cinq occurrences du vocable « eau » comme tel (4.59\%, pour la fréquence relative), il s'associe à plusieurs représentants du champ sémantique de l'eau: «ruissellent ", avec deux occurrences (1.83\%) et avec une occurrence $(0.92 \%)$ chacun: «bouillonne», «écoule», «île», «îles», «liquide», «onde», « ruisseler», « ruisselle» et «source». Au total, la fréquence relative de l'ensemble de ces vocables atteint près de $15 \%$. Ce champ sémantique pourrait être impliqué dans une symbolique de la vie ; « eau claire » et « eau vive», peut-on lire en effet dans le texte. L'étude de poèmes successifs montre que la présence de l'eau se résorbe: dans le poème intitulé Un mort demande à boire, l'eau vient à manquer.

Le mot-thème de la cinquième suite est «feu», et le troisième et dernier poème de cette suite est marqué par cet élément. Fièvre, en l'occurrence, contient les vocables «fièvre » et «feu », mais aussi «chaleur», «débris», «chauffe», «brouillant», «brûle», «flamme», «monte», «brouille», «brûlant», "cendres», «étouffent » et «intolérable». En déconstruisant «brouillant » et «brouille», c'est-à-dire en faisant sauter la lettre « $r »$, on découvre, dans le halo isotopique de la température élevée (la fièvre), des variantes flexionnelles du verbe «bouillir». La flamme s'imprime même sur le signifiant «oriflamme», qui présente une occurrence dans le poème.
Giguère (1973) a étudié l'œuvre de Garneau en relation avec la rêverie bachelardienne sur les éléments de la nature; selon lui, les éléments « chers » à ce poète sont l'air, l'eau et la terre. Vigneault (1980), en faisant référence au travail de Giguère, écrit que « tous les "éléments" seraient représentés dans l'œuvre de Garneau, sauf le feu » (p. 954). Or, SATO appliqué sur Regards et jeux dans l'espace aide à mettre en évidence que, d'une façon sectorisée, à savoir dans le lexique d'un poème, le feu, à l'instar d'ailleurs de l'eau, peut dominer.

\section{Vers une analyse plus fine du lexique: les thèmes de la vie et de la mort}

Nous avons illustré brièvement comment nous avons progressivement approfondi l'étude du lexique en allant de la surface des signes vers des regroupements en champs lexicaux ou sémantiques dès qu'une fréquence d'occurrences attirait notre attention. De façon plus structurée, nous avons exploré les réseaux de significations par la prise en considération de catégories cognitives. Pour cela, nous avons exploité la possibilité qu'offre SATO d'accoler aux formes hors contexte et aux occurrences en contexte des valeurs de propriétés diverses qui jouent le rôle d'interprétants au sens de Peirce (voir Deledalle 1979). C'est nous qui avons attribué les valeurs de propriétés selon nos hypothèses et nos intuitions, nous servant du logiciel SATO comme d'un assistant à une lecture et à une analyse personnalisées.

\section{Les thèmes de la vie et de la mort}

Le dénégatif, le privatif, cette « vie impossible» dont parle Hénault et le motthème dominant le recueil entier, «mort», sont des motifs qui nous ont poussés à nous intéresser aux thèmes de la vie et de la mort. Dès l'incipit du recueil, qui peut établir des "conditions de lisibilité" comme l'ont mis en évidence des sociocritiques (voir Angenot 1979), le lecteur se heurte à l'inconfort, au malaise et au verbe «mourir », puis à la vivacité et au tumulte du torrent:

$$
\begin{gathered}
\text { Je ne suis pas bien du tout assis sur cette } \\
\text { [chaise } \\
\text { Et mon pire malaise est un fauteuil où } \\
\text { [l'on reste } \\
\text { Immanquablement je m'endors et } \\
\text { [j'y meurs. } \\
\text { Mais laissez-moi traverser le torrent } \\
\text { [...]. (p. 9) }
\end{gathered}
$$

\section{Catégories pour le classement du vocabulaire de la vie et de la mort}

La thématique, selon Rastier (1989, 54-59), comprend deux grandes catégories de thèmes. D'une part, les thèmes génériques (ou classes sémantiques) lexicalisés peuvent être subdivisés en taxèmes ou thèmes microgénériques (cigarette, cigare, pipe, par exemple, s'opposent au sein du taxème //tabac//), en domaines ou thèmes mésogénériques qui incluent plusieurs taxèmes (comme mar. pour marine, cuis. pour cuisine) et en dimensions ou thèmes macrogénériques (//animé// vs /linanimél/, //animal// vs //humain//, //animél/ vs //végétal//). D'autre part, les thèmes spécifiques liés à une récurrence de sèmes -- ou unités minimales de sens -spécifiques peuvent exister indépendamment de toute lexicalisation (par exemple: sème /vulgarité/ ou sème /intensité/ présents dans plusieurs lexèmes, syntagmes et énoncés de L'Assommoir de Zola, ou bien regroupement de sèmes/chaud/, /visqueux/, /jaune/, et /néfaste/ dans des mots comme jus, pipi, sauce, morve, beurre, bedon, etc., dans la même œuvre).

En nous inspirant de ces subdivisions, nous avons d'abord étudié le thème "microgénérique " de la mort à travers le taxème qui rassemble en une catégorie ses divers représentants lexicalisés comme «mort(s)", «morte(s), «mortel», «mourir ", «meurs » ou « meurt " et «périr ».

Puis en prenant appui sur des lectures préliminaires du corpus, notamment celles des poèmes intitulés Les ormes et Pins à contre-jour, et en tenant compte de certains résultats quantitatifs, par exemple, que "saules » est l'unité dominante expliquant l'une des distances, nous avons librement "opérationnalisé" le concept de vie (ce lexème ne présente que trois oc- 
currences dans le recueil) à un niveau macrogénérique avec les règnes animal et végétal. Le Nouveau Petit Robert (c1993, 1996) définit les règnes comme les «grandes divisions de la nature, selon l'ancienne classification de Linné». Le Petit Larousse illustré définit "règne», dans son sens relatif aux sciences de la vie, comme "chacune des grandes divisions du monde vivant». L'édition 1998 de ce dernier dictionnaire ajoute: «Aux deux règnes traditionnels, animal et végétal, on substitue actuellement une répartition en cinq règnes: bactéries, protistes, champignons, plantes et animaux. " Pour nos fins d'exploration des possibilités du logiciel, nous nous sommes limités aux deux règnes traditionnels.

Les règnes pourraient ainsi être catégorisés plus finement selon plusieurs facettes: forme ou apparence externes («enfant(s)», «oiseau(x)», «bec», «fleur(s)»); forme ou attribut internes («os », «âme»); manifestation (“chanter», "roucoule») ou produit dérivé («cubes de bois»). Pour la démonstration, nous n'avons retenu que les deux premières facettes. Nous avons scindé, d'autre part, la catégorie animale en animal et humain.

Nous avons d'abord catégorisé le lexique hors contexte. Cela a suffi pour le type de traitement que nous avons fait subir au taxème de mort. Mais de façon à pouvoir "désambiguïser" certains lexèmes, en tenant compte aussi des déplacements de sens qui caractérisent la littérature et surtout la poésie, nous avons constaté qu'il s'avérait nécessaire de réaliser l'opération en contexte - possible dans SATO - pour notre traitement spécifique du thème de la vie.

\section{Analyse des résultats de la catégorisation}

Une fois que les valeurs de propriétés ont été distribuées, une analyse globale a été réalisée et la proportion de lexèmes marquant la vie et la mort dans chaque subdivision a été examinée. Le tableau 1 présente les résultats relatifs au taxème de mort et le tableau 2 ceux se rapportant à notre traitement du thème de la vie. La catégorie humain domine, suivie des catégories végétal, puis animal. Le nombre le plus élevé des occurrences représentant la mort se retrouve dans la sep-

Tableau 1. Nombre d'occurrences et pourcentages du lexique associés au taxème de mort suite à une catégorisation hors contexte

\begin{tabular}{|lccc|}
\hline \multicolumn{1}{|c|}{ Suite } & $\begin{array}{c}\text { Occur- } \\
\text { rence(s) }\end{array}$ & $\begin{array}{c}\text { Pourcentage } \\
\text { (suite) }\end{array}$ & $\begin{array}{c}\text { Occurrences cumu- } \\
\text { lées du corpus }\end{array}$ \\
\hline 1. Jeu \$ (5 poèmes) & 1 & $0.12 \%$ & 850 \\
\hline 2. Enf \$ (2 poèmes) & 0 & $0 \%$ & 1,108 \\
\hline 3. Esq \$ (7 poèmes) & 0 & $0 \%$ & 1,571 \\
\hline 4. Deu \$ (2 poèmes) & 10 & $2.02 \%$ & 2,067 (milieu) \\
\hline 5. Gri \$ (3 poèmes) & 6 & $1.26 \%$ & 2,543 \\
\hline 6. Fac\$ (3 poèmes) & 1 & $0.18 \%$ & 3,112 \\
\hline 7. San\$ (5 poèmes) & 11 & $0.99 \%$ & 4,221 \\
\hline Con \$ (poème de conclusion) & 0 & $0 \%$ & \\
\hline TOTAL & 29 & $0.66 \%$ & \\
\hline
\end{tabular}

tième suite, la même où s'inscrit le plus grand nombre d'occurrences se rapportant à la catégorie humain. Ce genre d'information peut être considéré comme une piste pour l'interprétation. Le végétal présente plus du double des occurrences animales, et c'est même lui qui reçoit le plus grand pourcentage dans une suite en particulier $(7.56 \%)$. À remarquer que dans cette suite, la troisième, les deux seules occurrences liées à la catégorie humain («tête», "chevelure») sont associées, par la métaphore ou dans un contexte d'anthropomorphisme, au végétal: "Les saules au bord de l'onde / La tête penchée / Le vent peigne leurs chevelures longues". (p. 16)

Le vocabulaire de la vie caractérisé par les règnes peut se regrouper en divers ensembles de relations. Le vocabulaire de l'humain, de l'animal et du végétal peut ainsi se rapporter à un tout: «paysan», «chevaux», «saules», «fleurs(s)» ou à une partie: «doigt(s)», «ailes», «branche(s)», «pollen»; à un élément pris isolément: «arbre(s)», «feuille(s)», «fleur(s) » ou à un regroupement de ceuxci : «forêts», «feuillage(s)», «bouquet»; puis à une classe d'objets générique: "bête(s) », « oiseau $(x)$ », «arbre(s)", «fleur(s)" ou à un objet spécifique: «oiseaux-mouches", "colombes", «ormes», «nénuphars». II est intéressant de constater que les occurrences de la classe générique sont numériquement plus importantes que celles de l'objet spécifique pour le vocabulaire de l'ornithologie. Cette proportion n'est pas stable pour le vocabulaire de la botanique. Ainsi, " oiseau $(x)$ " présente 12 occurrences, " oiseaux-mouches » et «colombes », 4 occurrences pour les deux vocables: il y a ici une certaine tendance à employer un vocabulaire simple et dépouillé. "Oiseaux" apparaît comme mot-thème dans le lexique de deux suites; un enfant est associé, dans le poème Portrait, à un oiseau, et c'est un oiseau qui à la fin «ne pourra s'en aller» (p. 34) qu'après avoir mangé le cœur du poète, avec l'âme de celui-ci au bec. Le terme «arbre(s) », lui, est présent 6 fois, mais nous avons 11 occurrences se rapportant à diverses espèces d'arbres: orme, pin, saule et tremble. Par contre, on retourne à la proportion de 9 occurrences pour «fleur(s) » contre 4 de «nénuphars ». Voilà qui illustre des variations dans la symbolique travaillant les représentants de la vie.

Grâce à une fonction de SATO, nous avons pu examiner les formes lexicales qui cooccurrent avec les représentants du taxème de mort, dans un contexte numérique de cinq lexèmes avant et après ceux-ci, en nous limitant aux noms communs, verbes et adjectifs. Avec un regroupement pour la fouille des 29 occurrences du taxème, «demande " (7 occurrences) 
Tableau 2. Nombre d'occurrences et pourcentages du lexique associés aux divisions du monde vivant en catégories humain $(H)$, animal $(\mathrm{A})$ et végétal $(\mathrm{V})$, suite à une catégorisation en contexte

\begin{tabular}{|c|c|c|c|c|c|c|c|}
\hline \multirow{2}{*}{ Suite } & \multicolumn{3}{|c|}{ Occurrence(s) } & \multicolumn{3}{|c|}{ Pourcentages (selon la suite) } & \multirow{2}{*}{ Total } \\
\hline & $\mathbf{H}$ & A & V & $\mathbf{H}$ & $\mathbf{A}$ & $\mathbf{V}$ & \\
\hline 1. Jeu $\$$ & 20 & 1 & 5 & $2.35 \%$ & $0.12 \%$ & $0.59 \%$ & $3.06 \%$ \\
\hline 2. Enf\$ & 7 & 3 & 1 & $2.71 \%$ & $1.16 \%$ & $0.39 \%$ & $4.26 \%$ \\
\hline 3. Esq\$ & 2 & 4 & 35 & $0.43 \%$ & $0.86 \%$ & $7.56 \%$ & $8.85 \%$ \\
\hline 4. Deu\$ & 22 & 3 & 17 & $4.44 \%$ & $0.60 \%$ & $3.43 \%$ & $8.47 \%$ \\
\hline 5. Gri\$ & 2 & 3 & 4 & $0.42 \%$ & $0.63 \%$ & $0.84 \%$ & $1.89 \%$ \\
\hline 6. Fac $\$$ & 7 & 1 & 0 & $1.23 \%$ & $0.18 \%$ & $0.00 \%$ & $1.41 \%$ \\
\hline 7. San\$ & 46 & 14 & 3 & $4.15 \%$ & $1.26 \%$ & $0.27 \%$ & $5.68 \%$ \\
\hline Con\$ & 4 & 0 & 0 & $2.38 \%$ & $0.00 \%$ & $0.00 \%$ & $2.38 \%$ \\
\hline TOTAL & 110 & 29 & 65 & $2.50 \%$ & $0.66 \%$ & $1.48 \%$ & \\
\hline $\begin{array}{l}\text { Pourcentages sans } \\
\text { les lexèmes sans pro- } \\
\text { priété }\end{array}$ & $54.19 \%$ & $14.29 \%$ & $32.02 \%$ & & & & \\
\hline
\end{tabular}

et «boire» (4) se sont avérés être les unités les plus denses disséminées autour du taxème, abstraction faite de la cooccurrence de deux représentants de ce dernier. Ce «halo textuel» de la mort renferme donc le sens de la survie, de la volonté de vivre; un vocabulaire lié à la vie à la demande et au besoin physiologique - côtoie en fait le taxème de mort. Compte tenu de cela ou de l'unité dominante expliquant toutes les distances calculées, en l'occurrence «joie", nous ne pouvons qu'être d'accord avec Blais (1973) lorsqu'il écrit dans son étude sur Garneau et le mythe d'Icare:

L'on s'est plu à mettre en relief la vocation de Saint-Denys Garneau pour la nuit, la douleur et la mort. Une tendance récente est d'insister sur sa vocation pour la vie, la lumière et la joie. Ces deux interprétations sont justes à la fois, et rendent compte de l'alternance incessante entre la face obscure et la face lumineuse de cet être ambivalent. (p. 17)

\section{Tentative d'analyse sémique}

Les regroupements en classes génériques ont contribué à rendre plus évidentes des concentrations dans certains poèmes ainsi qu'une évolution de la thématique de la vie qui reflue, à mi-chemin du recueil, à partir de la suite Deux paysages (voir le tableau 2). Les manifestations lexicales du thème de la mort sont entassées (neuf sur dix) dans le deuxième et dernier poème de la suite, Un mort demande à boire. Globalement, elles sont cependant peu nombreuses, si l'on s'en tient à l'étude du taxème (voir le tableau 1). Elles sont carrément absentes des derniers poèmes, alors que, par intuition, nous savons que ceux-ci sont empreints d'une atmosphère funeste que même la forme des mots contribue à évoquer par le rétrécissement de leur longueur. C'est pourquoi nous avons emprunté le mouvement inverse : plutôt que de procéder à des structurations autour de thèmes génériques, nous avons tenté une analyse microsémantique. Rastier (1989) a montré que la thématique s'exprime aussi par des récurrences de sèmes spécifiques indépendamment de lexicalisations déterminées.
Nous avons donc procédé à un codage en contexte de tout le vocabulaire du recueil dans lequel nous pouvions isoler des sèmes de vie et de mort ${ }^{10}$. Pour réduire la subjectivité inévitable d'une telle opération, nous avons au préalable élaboré une grille de sèmes codifiés à partir du contenu des entrées du dictionnaire Robert électronique des lexèmes "vie" et "mort». Cette démarche est conforme à l'affirmation de Greimas $(1986,83)$ selon lequel "on peut admettre que l'analyse des définitions nous renseignera sur la nature des sèmes (sinon de tous, du moins d'un certain nombre d'entre eux) implicitement contenus dans la dénomination". Dans des lexèmes comme "cendre", "douloureux", "étouffe", "glaciale", "guerre", «plainte", «fièvre", on peut facilement reconnaître des sèmes de la première catégorie, alors que «clair», «lu-

10. Par exemple, pour mort nous avons retenu : $M 1$ vieillesse; $M 2$ maladie, malaise; $M 3$ destruction, agonie, perte, deuil, disparition, décès; $M 4$ repos, repos éternel, sommeil, immobilité ; $M 6$ rigidité, etc. et nous avons catégorisé, pour vie: V1 enfance, origine, aube, printemps; V2 santé, vigueur; V3 construction, reconstruction; V4 lumière, clarté, transparence; V5 mouvement, animation, dynamisme, etc. 
mière », « désir », «danse », « riant », «transparence», «couleurs», etc., contiennent ceux qui sont habituellement associés à la vie, bien qu'il faille apporter des nuances.

Cependant, tous les lexèmes ne s'analysent pas aussi aisément; il faut tenir compte non seulement des mots simples, mais également des mots composés, des syntagmes et des effets d'inhibition de sèmes opposés dans des séquences (que l'on pense à l'expression «petits monstres»), accepter l'impossibilité de l'exhaustivité, composer sans arrêt entre désir de systématicité et intuition, concilier sèmes potentiels dans le lexique et sèmes effectivement réalisés dans le texte, connotations personnelles et effets contextuels. Si l'on reconnaît que le texte, en particulier le texte poétique, travaille bien à ce niveau microsémantique sur le lecteur, il est lourd de décomposer les processus en jeu et sans doute artificiel de vouloir les contrôler. Probablement faudrait-il accepter la codification de deux lectures distinctes par deux lecteurs aux connaissances et aux sensibilités différentes. Bref, l'ampleur de la tâche et les divergences des deux codeurs nous ont dissuadés de mener l'expérience jusqu'au bout.

\section{Le texte littéraire et la pluralité de son approche}

Le texte littéraire peut être envisagé sous de multiples facettes: ses lectures ou ses approches critiques peuvent s'appuyer sur plusieurs substrats théoriques qui permettent d'en articuler diverses interprétations. Dans leur Manuel bibliographique des études littéraires, Beugnot et Moureaux identifient diverses voies de la recherche littéraire, à savoir l'histoire et les genres littéraires, la poétique, l'imaginaire, la littérature et la psychanalyse, la sociologie et littérature, etc. Pour leur part, Bergez et ses collaborateurs, dans leur Introduction aux méthodes critiques pour l'analyse littéraire, parlent de critique génétique, psychanalytique, thématique et textuelle ainsi que de sociocritique. Quant aux rapports entre littérature et cognition, ils font l'objet de toute une partie de l'ouvrage, publié sous la direction de Claude Duchet et Stéphane Vachon et intitulé La recherche littéraire: objets et méthodes. Après avoir illus- tré les explorations thématiques rendues possibles par diverses catégorisations, nous allons maintenant proposer quelques pistes d'utilisation de SATO, d'abord pour caractériser le style, puis pour aider l'analyse sociocritique ainsi que des approches d'inspiration psychanalytique et intertextuelle.

\section{Quelques indicateurs du style}

Difficile que de définir le texte littéraire dans sa complexité, sa symbolique, sa surdétermination. L'étude de la thématique ne suffit pas, même si plusieurs niveaux de généricité et de spécificité sont pris en compte. Les théoriciens pourraient en dire bien long sur le sujet mais ce n'est pas là notre projet. Mentionnons simplement que le texte littéraire est aussi caractérisé par le style, dont il est possible de trouver plusieurs indicateurs. Nous avons notamment relevé une tendance de SaintDenys Garneau à employer un vocabulaire simple et dépouillé par l'usage privilégié de la classe générique par rapport à l'objet spécifique dans le champ de l'ornithologie. Cette tendance semble aussi se confirmer avec l'utilisation de lexèmes plutôt courts, comme nous l'avons constaté plus haut, et ce, même pour des mots non fonctionnels très apparents dans le lexique trié par ordre de longueur décroissante des formes: «eau» (15) ${ }^{11}$, «air » (8), «feu » (8), «mer» (7), «âme» (3), «fin » (3), «mur» (3), « os » (3), «vie» (3), etc. Pour l'ensemble du lexique global, la proportion des mots de trois et de quatre lettres est respectivement de $17 \%$ et de $16 \%$, chiffres beaucoup plus importants que ceux que nous avons obtenus avec toutes sortes de textes non littéraires traités lors de nos expérimentations antérieures. Le pourcentage le plus élevé de mots de deux, trois et quatre caractères se situe dans la deuxième moitié du recueil. Et comme l'indique un tri par catégories grammaticales, le poète fait usage d'adjectifs relativement simples: «seul» (moins de 10 occurrences), «claire» (8), «froid» (moins de 5 occurrences), «longues» (4), «petit» (4), «grande» (3), etc. La ponctuation, aussi, est réduite (seulement 64 points). Cela nous a portés à faire un rapprochement avec la nature (le style) «squelettique» du texte de Garneau que nombre de critiques ont remarqué12, et qui peut prendre appui sur ce vers que nous avons déjà cité précédemment: « Je suis une cage d'oiseau / Une cage d'os" (p. 33). Bien entendu nous faisons le lien entre squelette et le thème de la mort. À propos du dessein de l'œuvre du poète, Marcotte (1969) écrivait en outre: "de la fluidité de l'eau à l'irréductible dureté des os». Le Dictionnaire des symboles de Chevalier et Gheerbrant (1982) signale: "L'usage d'os humains en Indes et au Tibet pour la confection d'armes divines ou d'instruments de musique n'est pas étranger à ces considérations: ascèse, dépassement de la notion de vie et de mort, accès à l'immortalité». (p. 712)

La dualité, l'opposition comme la symétrie appartiennent au style de Garneau et cela nous a incité à examiner la fréquence du chiffre «deux». II revient à 14 reprises $(0.32 \%)$, dont 10 fois $(2.02 \%)$ dans la suite Deux paysages et 9 (3.90\%) dans son premier poème, quinzième des 28 poèmes du recueil, à savoir Paysage en deux couleurs sur fond de ciel. $\mathrm{Ce}$ poème met en scène, de façon géométrique et schématique, notre thématique: "La vie la mort sur deux collines / Deux collines quatre versants" (p. 19). En considérant les fréquences cumulées, cette suite intitulée Deux paysages coïncide à peu près avec le milieu du recueil (la 2197 eccurrence).

\section{Aide à l'analyse sociocritique}

Le lexique du poème Accueila retenu particulièrement notre attention parce qu'il rassemble, dans les années trente au Québec, deux isotopies: l'une religieuse («Dieu», «accueil ", «dons", "grâce ", «parfaits», « recueillement», etc.), l'autre féminine («fleur», «exquise», "gazelle», "grâce", "pudeur", etc.). Ce poème où le sens se met à trembler, pour faire allusion à Barthes - $a$ fait couler beaucoup d'encre parmi les critiques. Entre autres, Vigneault (1973) y voit l'amour de la femme s'associer à un idéal mystique. En

11. Les occurrences sont entre parenthèses.

12. Beaudoin (1993) parlera d'une «maigreur squelettique» hantant le vers de Garneau. «...dépouillé jusqu'à l'os», écrit Trottier (voir Nepveu 1988). «...l'os ou la présence dedans/dessous la blessure», renchérit Gagnon (1982) 
fait, il semble s'opérer dans ce petit texte une sorte de travail idéologique où la voix du religieux se lie à celle du féminin. On pourrait avancer que Accueil tend à reproduire, tout en les décentrant ${ }^{13}$, des éléments du discours social des années trente qui avaient dû être médiatisés par la mystique du chanoine Lionel Groulx et par la morale de l'époque. Sur le plan des idées, il faut rapprocher l'œuvre de SaintDenys Garneau de la revue La Relève, dans laquelle l'écrivain a publié quelques textes (voir Saint-Denys Garneau et La Relève 1995).

La question du vers libre utilisé par Garneau pourrait aussi être traitée à la lumière de l'assertion de Biron (1995) selon laquelle «l'alexandrin signifie l'ordre social» (p. 129). Garneau se serait dissocié, mais pas tout à fait, de la versification. Comme le fait remarquer Lemaire (1994), parmi le «prosaïsme [de Regards et jeux dans l'espace, on] reconnaît ici le rythme de l'alexandrin: ["Un enfant est en train de bâtir un village"]» (p. 81). Dans ce contexte et en tenant compte de la dualité caractéristique dont nous avons parlé, puis en ayant en tête la distribution des thèmes de la vie (la végétation ...) et de la mort, le recueil nous est apparu comme une gestalt plus ou moins analogue à un alexandrin, avec un hémistiche de vie, un second de mort, et une césure initiée par la suite Deux paysages. Avant la césure, les "ormes calmes" «...font de l'ombre légère / Bonnement / Pour les bêtes" (p. 17), et après celle-ci, "Les bêtes ont les yeux effarés" (p. 24), la nuit est lâchée sur terre... «Quand on sait ce que c'est / Quelle bête c'est» (p. 27) ${ }^{14}$. Marcotte (1969) note aussi que, dans les années trente au Québec, «le vers libre signifie le rejet d'une tradition extrêmement puissante, d'un ordre littéraire» (p. 40). En plus de participer à un style «squelettique " de l'œuvre ou à une certaine nature aérée, voire aérienne, de celle-ci, la ponctuation limitée (90 signes pour 4395 occurrences) et utilisée librement se rattacherait à cette signification sociale du vers libre.

D'autre part, le discours social ou "sociogramme" de cette décennie marquée par la crise invite à un retour à la terre pour une fuite hors de la ville (voir Popovic, dont parle Benoît Melançon 1994). En référence à ce discours, notre analyse thématique de la vie et de la mort pourrait être relancée quand on lit ce passage d'une lettre de Garneau: «Et je hais la ville, toute cette mort ambulante [...] tandis qu'ici [en cette campagne], il n'est rien qui vive, qui ne remue». (ibid., p. 102)

\section{Aide à l'analyse d'inspira- tion psychanalytique}

En face du texte littéraire, si la sociocritique essaie de mettre en évidence des rapports avec la société, la lecture d'inspiration psychanalytique tente, pour sa part, de faire des liens avec l'appareil psychique ou le sujet désirant.

Nous pouvons faire de nouveau référence aux catégories humain, animal et végétal pour étudier le vocabulaire d'objets présentés globalement ou de manière intégrée et ceux présentés de façon fragmentée ou morcelée, et cela en rapport avec le poème de conclusion Accompagnement. Ce poème, qui vient à la suite d'une dramatisation de repliement sur soi ${ }^{15}$, met en scène une sorte de dissociation psychique ${ }^{16}$ ou de division de l'être ${ }^{17}$ :

\section{fe marche à côté d'une joie \\ D'une joie qui n'est pas à moi \\ [...] \\ Mais je machine en secret des échanges \\ Par toutes sortes d'opérations, des \\ [alchimies, \\ [...] \\ Afin qu'un jour, transposé, \\ Je sois porté par la danse de ces pas de joie \\ Avec le bruit décroissant de mon [pas à côté de moi \\ Avec la perte de mon pas perdu \\ s'étiolant à ma gauche \\ Sous les pieds d'un étranger \\ qui prend une rue transversale. (p. 34)}

Les 99 parties que nous avons pu identifier: "bec», «branches», "chevelure», «épaule», «feuille», «fleur», «os», «paupières», «pied», «tête», «visage», "yeux», etc. peuvent apparaître comme des compléments, dans une proportion relativement harmonieuse ${ }^{18}$, avec les 80 occurrences d'intégration d'objet dans une forme globale: «arbre», «baigneuse», "colombes», «enfant», «épinette», «mouche», «oiseau», «paysan», «poisson », "saule», «servantes», etc.

Les thèmes de la vie et de la mort peuvent-ils être reliés à Éros et Thanatos, c'est-à-dire les instincts de vie et de mort qui s'imbriquent l'un l'autre dans la psyché ? En ce qui a trait à Éros, l'animalité d'une production symbolique textuelle, son côté «bestiaire», pourrait être analysé en fonction de son intensité, cela proportionnellement aux représentants humain. Et on pourrait analyser la qualité de la matière instinctuelle symboliquement mise en œuvre, en catégorisant divers représentants animal. Par exemple, face à ce vers de Garneau: "Où la gazelle de votre grâce évoluera» (p. 33), nous pourrions associer à «gazelle » l'étiquette courtois ou sublime, s'opposant à pulsionnel ou archaïque, que l'on pourrait accoler à des représentants animal comme ceux de ces vers du poème Le Léthé de Baudelaire: «Tigre adoré, monstre aux airs indolents; / Je veux longtemps plonger mes doigts tremblants / Dans l'épaisseur de ta crinière lourde» (p. 215). Pour un corpus en particulier, il faudrait d'abord évaluer la pertinence et la faisabilité de ce type d'analyse rejoignant la dimension expressive du style.
13. Ce genre de phénomène est beaucoup étudié en sociocritique, où l'analyse du discours s'enracine dans la «matérialité du langage» (CIADEST 1993), et notamment dans les données lexicales (voir, par exemple, Robin 1993).

14. À remarquer au passage, l'utilisation par le poète des formes homophones «sait» et «c'est».

15. L'appareil psychique peut ici être un ancrage. Nous avons vu que le paysage du début du recueil cède la place à une maison fermée et que l'unité lexicale expliquant la distance de la deuxième suite était tournée vers l'extérieur, à savoir le pronom de la troisième personne, «il », tandis que les pronoms de la première personne prennent beaucoup de place dans les deux dernières divisions. Garneau va jusqu'à écrire vers la fin du recueil: «'J'ai gô̂té à la fin $d u$ monde». (p. 31)

16. La deuxième topique freudienne (les instances du ça, du moi et du surmoi) relative à l'appareil psychique pourrait ici être un ancrage théorique, et la relation entre les instances psychiques peut être prise en compte pour essayer de comprendre le processus créateur. Le genre d'informations traitées dans cette section de l'article peut se rencontrer aussi en psychologie projective.

17. «Division de l'être», si l'on se positionne plus du côté existentiel ou de l'expérience métaphysique. Ainsi que le fait remarquer Chemla (1994), «SaintDenys Garneau envisage la poésie comme la voie de connaissance privilégiée d'une quête morale et spirituelle, animée par un tourment métaphysique et religieux». Par rapport au «constat d'échec d'Accompagne$m e n t »$, ce même auteur écrit : «plus le poète cherche à investir la réalité par le regard et le jeu, plus se creuse en lui la profondeur de l'exil, du silence et de la mort».

18. Cette proportion pourrait être liée à la représentation d'une manifestation de mécanismes perceptuels ou de processus cognitifs. 


\section{Aide à l'analyse intertextuelle}

"Créer par ingéniosité un espace analogue à l'Au-delà..." (p. 27), voilà l'aspiration du poète Garneau qui a lu Baudelaire (Bourneuf 1969). II est possible de cerner plus d'un rapport d'intertextualité avec l'auteur des Fleurs du mal. $\mathrm{Ce}$ dernier, dans Le peintre de la vie moderne, écrit:

[...] le génie n'est que l'enfance retrouvée à volonté, l'enfance douée maintenant, pour s'exprimer, d'organes virils et de l'esprit analytique qui lui permet d'ordonner la somme des matériaux involontairement amassée. C'est à cette curiosité profonde et joyeuse qu'il faut attribuer l'oil fixe et animalement extatique des enfants devant le nouveau, quel qu'il soit, visage ou paysage, lumière [...] (p. 888)

Dans cette définition du génie aux allures de poétique, on retrouve ensemble «enfance», «joyeuse», «œil », «enfants", "paysage " et «lumière » qui appartiennent au lexique, à l'univers de Saint-Denys Garneau (aussi peintre à ses heures).

Dans Regards et jeux dans l'espace, «enfant(s)" présente 12 occurrences; «œil », 1 occurrence; “yeux», 18; «regard(s) », 10 et « regard » tronqué, 15 ; « paysage(s)", 6 ; «lumière(s) », 10 ; «lueurs », 1 ; "luira", 1 ; "lumineuses", 1 ; «clair » tronqué, 11 et "clarté», 3 , etc., sans parler de «joie». Nous avons adressé à SATO une série de requêtes pour obtenir toutes les occurrences selon leur distribution par rapport aux suites de poèmes. Entre autres, 5 des 8 «claire» sont concentrés dans la suite Esquisses en plein air, d'où une manifestation encore de la cohérence textuelle (nous ne sommes pas ici dans un surréalisme débridé); pour ce qui est des 7 occurrences "enfant", elles se disséminent ainsi dans les suites: 2 dans Jeux, 1 dans Enfants, puis 4 dans Sans titre.

De Baudelaire à Garneau, cette grappe (cluster) a essaimé, pourrait-on penser, mais en se réorganisant. $\grave{A}$ la suite de notre catégorisation sémantique où, quantitativement selon les occurrences, le végétal ressort deux fois plus que l'animal, et où proportionnellement la densité dans une suite est de loin la plus forte pour le végétal $(7.56 \%)$, nous sommes, en effet, tentés de voir le génie de notre poète plus animé par un enfant à l'oeil « végétalement " extatique que "animalement extatique $" . .$.

\section{Commentaires sur l'utilisation des résultats}

En fait, les mêmes approches méthodologiques, à savoir l'étude statistique des occurrences et des cooccurrences de signes purement graphiques ou de phénomènes issus d'une catégorisation du vocabulaire en contexte ou hors contexte, peuvent sous-tendre des interprétations multiples dans la mesure où le logiciel permet une souplesse de catégorisation et de découpage. En effet, il est possible de faire varier la nature des unités linguistiques (vocables, lexèmes, unités terminologiques simples ou complexes), de les décomposer en unités minimales de forme (morphèmes, comme les suffixes, préfixes, etc.) ou de sens (sèmes) et de les regrouper en catégories linguistiques, sémantiques ou cognitives de granularité variée. L'attribution des catégories peut être effectuée en référence à la sémiotique, à la sociocritique ou à la psychanalyse, entre autres. En outre, l'observation peut porter sur des unités textuelles de longueur et de types divers. On pourrait tout aussi bien prendre le vers, l'hémistiche, la phrase, le paragraphe ou d'autres éléments pour des études stylistiques que l'œuvre complète, les parties de l'œuvre (poèmes et divisions, chapitres, etc.), les œuvres d'un même auteur, d'une même période, d'un même genre littéraire, le corpus de deux ou de plusieurs auteurs. La voie est alors ouverte à toutes sortes de comparaisons dans le cadre des diverses théories utilisées. II y a, par exemple, une parenté d'approche évidente entre certaines analyses intertextuelles et l'idéométrie liée aux champs de la connaissance; Bertrand $(1995,35)$ donnait l'exemple d'une « forte présence du thème associant la compassion pour la misère humaine à la dérision de l'ambition humaine dans la poésie anglaise entre 1550 et 1650 et dans la peinture hollandaise du XVIIe siècle". Floridi $(1996,12)$ trouve d'ailleurs que Internet, avec ses nombreux corpus de textes et de sources multimedias, devient le laboratoire idéal de l'idéométrie devenue le lieu de rencontre de plusieurs disciplines comme l'historiographie scientométrique, la lexicographie, la stylométrie, la prosopographie, l'analyse de citations, la bibliomérie, l'économétrie, etc. Cela rejoint les préoccupations des littéraires désireux d'utiliser l'informatique pour explorer des problématiques jusque-là inédites ou, à tout le moins, impossibles à étudier à grande échelle, dont nous faisions état plus haut. Par ailleurs, nous avons pu (BertrandGastaldy 1993) rendre opérationnelle l'intertextualité pour extraire des connaissances en vue de la mise au point d'un prototype de système expert d'aide à l'analyse documentaire de textes de jurisprudence.

Dans le cadre de recherches futures sur le recueil de Saint-Denys Garneau, nous pourrions le comparer à d'autres œuvres numérisées; les avantages du recours à l'informatique apparaîtraient alors plus clairement qu'ils n'apparaissent sur ce corpus d'envergure, somme toute, limitée. Nous pourrions aussi pousser plus loin les traitements statistiques. Des efforts supplémentaires de codage pourraient conduire à délimiter les vers ou à procéder à une catégorisation phonétique. II serait, pour cela, très enrichissant de recourir, pour le balisage, à la norme SGML et aux recommandations du TEI (Text Encoding Initiative) émises spécifiquement pour les œuvres poétiques, afin de vérifier dans quelle mesure il est possible de surmonter certaines limites inhérentes au logiciel SATO. En effet, parce que celui-ci est générique, il n'a pas été conçu pour tenir compte de certaines spécificités du texte littéraire, en particulier du texte poétique, comme le contrôle des différentes variantes du texte, le respect de la mise en page ou de la présentation du vers libre.

\section{Conclusion}

Bien que limitée, notre étude n'en a pas moins illustré différentes approches du texte littéraire à l'aide d'un logiciel qui permet d'enrichir et de modifier les analyses au fur et à mesure que naissent certaines intuitions, que s'infirment ou se vérifient certaines hypothèses. II offre non seulement des lectures supplémentaires du vocabulaire sur l'axe vertical qui s'ajoutent à la lecture linéaire traditionnelle, mais aussi des déconstructions et 
des reconstructions d'unités textuelles variées. Les décomptes, juxtapositions et comparaisons sont d'autant plus riches que peuvent être combinés les signes graphiques et leurs diverses propriétés, dont le lecteur peut en partie contrôler la nature et la granularité, en contexte ou hors contexte. Des analyseurs comme la distance, inaccessibles à l'humain, ont pu être appliqués pour comparer différents sous-ensembles du corpus.

Envisagés comme la source de possibilités accrues d'investigations, d'approfondissements et d'aides à l'analyse d'un ou de plusieurs corpus, les logiciels d'analyse de textes tels que SATO ne constituent pas une menace pour l'avenir des études littéraires. Dans la mesure où ils demeurent un appui aux capacités de traitement, de catégorisation et de mémorisation de la personne humaine, il n'y a pas lieu de s'alarmer de la déconstruction qu'ils engendrent. Béhart $(1994,16)$ écrit que "enfanté dans la douleur, le poème mérite le respect le plus absolu». Que l'acte créateur se soit réalisé dans la douleur ou la jubilation, nous acquiesçons quand il est question de respect du poème, cet objet esthétique, mais lorsque cet auteur affirme: "II n'est pas tolérable qu'il soit torturé, émasculé comme le fait la machine", nous refusons d'être aussi alarmistes. La culture personnelle et la pensée, le goût de la lecture et le plaisir du texte, pour employer le titre d'un essai de Barthes, demeurent. Ils sont à l'honneur, pour nous, avec cette vague de numérisation des documents.

Dans la recherche exploratoire que nous avons effectuée, nous avons voulu tester, en tant que spécialistes de la documentation et littéraires, les possibilités qu'offrent les nouvelles technologies de l'information et de la communication au personnel doté de cette double formation. Trop souvent, mais à juste titre, les littéraires ont baissé les bras devant l'inutilité de l'analyse-sujet fournie par les catalogues de bibliothèques. La sensibilisation aux besoins réels de clientèles spécifiques ainsi que l'évaluation et la maîtrise des logiciels disponibles devraient accroître la crédibilité de ce personnel et lui permettre une plus grande intégration au sein d'équipes d'enseignement et de recherche, universitaires notamment. Les spécialistes de la documentation peuvent, en effet, se tailler une place de choix dans ce grand mouvement d'évolution des bibliothèques tradi- tionnelles vers des bibliothèques virtuelles. Dans ce contexte, leur rôle n'est pas nécessairement limité à des fonctions, pourtant nouvelles et passionnantes, d'adaptation des normes de description bibliographique des textes électroniques, de développement de la compatibilité entre les notices bibliographiques en format MARC et les textes encodés selon le SGML (Guenther 1994), de modification des politiques de développement des collections tenant compte des particularités des nouveaux supports et des questions de droit d'auteur, entre autres (Gaunt 1995). Mais comme le prouve le rôle «proactif» que jouent les bibliothécaires de référence de l'Information Arcade à l'Université de l'lowa (Lowry 1994), il y a beaucoup de services nouveaux à développer pour assister les étudiants et même certains professeurs dans l'exploration et l'analyse de textes électroniques au moyen des logiciels; par exemple, la comparaison des divers logiciels disponibles ou l'évaluation de la façon dont ceuxci peuvent limiter, décupler ou infléchir les analyses effectuées traditionnellement «à la main ». Du point de vue de l'analyse documentaire, il est également intéressant de constater que la technologie ouvre la voie à une mise en perspective et à un élargissement des pratiques habituelles. De l'analyse du sujet à l'analyse littéraire assistées par ordinateur, plusieurs pas restent à franchir, certes, mais il existe une panoplie d'outils et une adaptation des méthodologies, comme le montrent bien nos différents travaux dans ces deux domaines avec un logiciel susceptible de répondre en partie à des objectifs distincts.

\section{Sources consultées}

Angenot, Marc. 1979. Glossaire pratique de la critique contemporaine. LaSalle (Qc): Hurtubise $\mathrm{HMH}$.

Baudelaire, Charles. 1954a. Les Fleurs du mal (1861). In: Oeuvres. Bibliothèque de la Pléiade. [Paris]: Gallimard, p. 77-245.

1954b). Le peintre de la vie moderne (1863). In: Oeuvres. Bibliothèque de la Pléiade. [Paris]: Gallimard, p. 881-920

Beaudoin, Réjean. 1993. Saint-Denys Garneau, hier et aujourd'hui. In: Regards et jeux dans l'espace de Saint-Denys Garneau (postface) Boréal compact classique; 51 . Montréal: Boréal, p. 83-106.

Béhart, Henri. 1994. Le traitement du plein texte. In: Traitements informatisés de corpus textuels.
Textes réunis par Éveline Martin. Études de sémantique lexicale. Paris: Didier Érudition, p.1329.

Bertrand, Guy. 1995. Des principes pour l'univers des inforoutes; un univers sans règles, sans standards, un milieu sans lois ni frontières. In: Documentation sans frontières? Comptes rendus $d u$ $22^{\circ}$ congrès de l'ASTED, p. 21-53.

Bertrand-Gastaldy, Suzanne et Gracia Pagola. 1992. L'analyse du contenu textuel en vue de la construction de thésaurus et de l'indexation assistées par ordinateur; applications possibles avec SATO (système d'analyse de textes par ordinateur). Documentation et bibliothèques 38(2): 75-89.

Bertrand-Gastaldy, Suzanne. 1993. Analyse documentaire et intertextualité. In Les Sciences du texte juridique: Le droit saisi par l'ordinateur, sous la direction de Claude Thomasset, René Côté et Danièle Bourcier. Cowansville: Les Éditions Yvon Blais, p. 139-173.

Bertrand-Gastaldy, Suzanne, avec la coll. de Gracia Pagola. 1994. Le contrôle du vocabulaire et l'indexation assistés par ordinateur: une approche méthodologique et un procédurier pour l'utilisation de SATO. $2^{\ominus}$ éd. revue et augmentée d'un index. Montréal: Université de Montréal, École de bibliothéconomie et des sciences de l'information.

Beugnot, Bernard et José-Michel Moureaux. 1982. Manuel bibliographique des études littéraires: les bases de l'histoire littéraire: les voies nouvelles de l'analyse critique. Paris: Nathan (NathanUniversité).

Biron, Michel. 1995. Desrochers: boucherie, terroir et idéologie. In Micellanées en l'honneur de Gilles Marcotte, sous la dir. de Benoît Melançon et Pierre Popovic. Montréal: Fides, p. 129-142.

Blais, Jacques. 1973. Saint-Denys Garneau et le mythe d'lcare. Sherbrooke: Cosmos.

Bourneuf, Roland. 1969. Saint-Denys Garneau et ses lectures européennes. Québec: Presses de l'Université Laval.

Chemla, Yves. 1994. Regards et jeux dans l'espace. In Dictionnaire des oeuvres littéraires de langue française, de Jean-Pierre de Beaumarchais et Daniel Couly. [Paris]: Bordas, p. 1658.

Chevalier, Jean et Alain Gheerbrant. 1982. Dictionnaire des symboles: mythes, rêves, coutumes, gestes, formes, figures, couleurs, nombres. Bouquins. Paris: Robert Laffont / Jupiter.

CIADEST. 1993. Le Centre interuniversitaire d'analyse du discours et de sociocritique des textes. Cahier de recherche; 1 . Montréal: CIADEST.

Daoust, François. 1996. SATO: Système d'analyse de texte par ordinateur: version 4.0: manuel de référence. Montréal: Service d'analyse de textes par ordinateur (ATO), Université du Québec à Montréal.

Deledalle, Gérard. 1979. Théorie et pratique du signe; introduction à la sémiotique de Charles S. Peirce. Paris: Payot.

Floridi, Luciano. 1996. Internet: which future for organized knowledge, Frankenstein or Pygmalion? The Information Society 12: 5-16.

Fortier, Paul A. 1995. Categories, theory, and words in literary texts. Research in Humanities Computing 5: 91-109. 
Gagnon, Jean. 1982. Saint-Denys Garneau: l'autour, les centres. Thèse de doctorat inédite. Montréal: Université de Montréal.

Garneau, Hector de Saint-Denys. 1971. Regards et jeux dans l'espace. In Saint-Denys Garneau: Oeuvres, texte établi par Jacques Brault et Benoît Lacroix. Montréal: Les Presses de l'Université de Montréal.

Gaunt, Marianne I., 1995. Literary text in an electronic age: implications for library services. Advances in Librarianship 19: 191-215.

Giguère, Richard. 1973. D'un «équilibre impondérable " à une "violence élémentaire": évolution thématique de la poésie québécoise 19351965: Saint-Denys Garneau, Anne Hébert, Roland Giguère et Paul Chamberland. Voix et images du pays VII: 51-90.

Greimas, Algirdas Julien. 1986. Sémantique structurale. Paris: Presses universitaires de France, 1986.

Guenther, Rebecca S. 1994. The challenges of electronic texts in the library: bibliographic and access. In Literary Texts in an Electronic Age; Scholarly Applications and Library Services. Edited by Brett Sutton. Urbana-Champaign, III. Graduate School of Library and Information Science, University of Illinois at UrbanaChampaign, p. 149- 172.

Guiraud, Pierre. 1975. Les caractères statistiques du vocabulaire. In: La stylistique: lectures, de Pierre Guiraud et Pierre Kuentz. Paris: Klincksieck, p. 222-224.

Hamm, Jean-Jacques et Gregory Lessard. 1993: Informatique et interprétation: présences et absences textuelles dans les romans de Stendhal. Texte: Revue de critique et de théorie littéraire 13-14: 263-274.

Hénault, Gilles. 1969. Saint-Denys Garneau ou la vie impossible. Études françaises 5 (4): 480-489.

Introduction aux méthodes critiques pour l'analyse littéraire. 1990. Sous la dir. de Daniel Bergez. Paris: Bordas.

Laffal, Julius. 1995. A concept analysis of Jonathan Swift's A Tale of a Tub and Gulliver's Travels. Computers and the Humanities 29: 339-361.

Lemaire, Michel. 1994. Métrique et prosaïsme dans la poésie de Saint-Denys Garneau. Voix et images: littérature québécoise 58: 73-84.

Lenoble, Michel. 1993. Une génération perdue Texte: Revue de critique et de théorie littéraire; 13-14: 47-58.
Lenoble, Michel et Conrad Sabourin. 1993. Informatique et littérature: panorama et évolution Texte: Revue de critique et de théorie littéraire 13-14: 307-322.

Literary Texts in an Electronic Age; Scholarly Applications and Library Services. 1994. Edited by Brett Sutton. Urbana-Champaign, III. : Graduate School of Library and Information Science, University of Illinois at Urbana-Champaign.

Lowry, Anita K. 1994. Electronic texts and multimedia in the academic library: a view of the front line. In Literary Texts in an Electronic Age; Scholarly Applications and Library Services. Edited by Brett Sutton. Urbana-Champaign, III. : Graduate School of Library and Information Science, University of Illinois at Urbana-Champaign, p. 57 66.

Marcotte, Gilles. 1969. Le temps des poètes: description critique de la poésie actuelle au Canada français. Montréal: $\mathrm{HMH}$.

Martin, Éveline. 1993. Reconnaissance de contextes thématiques dans un corpus textuel; éléments de lexico-sémantique. Paris: Didier Érudition.

1995. Thème d'étude, étude de thème. In L'analyse thématique des données textuelles; l'exemple des sentiments. Dirigé par François Rastier, publié par Éveline Martin. Paris: Didier Érudition, p.13-23.

Melançon, Benoît. 1994. Pour une lecture sociale de la correspondance de Saint-Denys Garneau. Voix et images: littérature québécoise 58: 96106.

Melançon, Robert. 1994. Journal, atelier, recueil. Voix et images: littérature québécoise 58: 26-40.

Nepveu, Pierre. 1998. L'écologie du réel: mort et naissance de la littérature québécoise contemporaine. Papiers collés. Montréal: Boréal.

Olivier, Andrew. 1993. Du bon usage des textes élec troniques: lesquels? Texte: Revue de critique et de théorie littéraire 13-14: 291-306.

Olsen, Mark. 1993. Signs, symbols and discourses: a new direction for computer-aided literature studies. Computers and the Humanities 27(5-6): 309-314.

Ouellet, Pierre. 1994. Le jeu du regard dans l'espace poétique de Saint-Denys Garneau. Voix et ima ges: littérature québécoise 58: 50-61.

Rastier, François. 1989. Sens et textualité. Langue, Linguistique, Communication. Paris: Hachette
Rastier, Francois, 1995. Avant-propos. In L'analyse thématique des données textuelles; l'exemple des sentiments. Dirigé par François Rastier, publié par Éveline Martin. Paris: Didier Érudition, p. 7-10.

La recherche littéraire: objets et méthodes. 1993. Sous la dir. de Claude Duchet et Stéphane Vachon. Montréal: XYZ; Paris: Centre de coopération interuniversitaire franco-québécoise; SaintDenis, France: P.U.F.

Robin, Régine. 1993. «Le sociogramme en question. Le dehors et le dedans du texte" et "Pour une socio-poétique de l'imaginaire social». In Discours social: analyse du discours et sociocritique des textes 5 (1-2): 1-15, 7-32.

Saint-Denys Garneau et La Relève. 1995. Sous la dir. de Benoît Melançon et Pierre Popovic. Nouvelles études québécoises. Montréal: FidesCétuq.

Smoje, Dujka. 1984-85. Saint-Denys Garneau: écrits sur la musique. Études françaises 20 (3) : 65-95

Taylor, Dennis. 1993. Literary texts and the state of the language: the role of the computer. Computers and the Humanities 27: 341-347.

Teil, Geneviève. 1994. Diversité et contrôle de la lecture: vers une aide à l'analyse de gros corpus de textes. In Traitements informatisés de corpus textuels. Textes réunis par Éveline Martin. Paris: Didier Érudition, p. 185-206.

Texte: revue de critique et de théorie littéraire, volumes thématiques intitulés «Texte et informatique». 1993. Toronto: Éditions Trintexte, vol. 13-14.

Vigneault, Robert. 1973. Saint-Denys Garneau à travers Regards et jeux dans l'espace. Montréal: Les Presses de l'Université de Montréal.

Vigneault, Robert. 1980. Regards et jeux dans l'espace. In Dictionnaire des oeuvres littéraires du Québec, sous la dir. de Maurice Lemire, avec la collab. de Gilles Dorion, André Gaulin et Alonzo Le Blanc, tome 2, 1900-1939. Montréal: Fides, p. 949-956

Vuillemin, Alain. 1993. Informatique et textes poétiques. Texte: Revue de critique et de théorie littéraire 13-14: 21-31

Wooldridge, Terence Russon. 1993. Le flou en informatique textuelle. Texte: Revue de critique et de théorie littéraire 13-14: 275-289. 